\title{
OBDD-Based Sectionalizing Strategies for Parallel Power System Restoration
}

\author{
Chong Wang, Student Member, IEEE, Vijay Vittal, Fellow, IEEE, and Kai Sun, Member, IEEE
}

\begin{abstract}
After a power system is subjected to a blackout, parallel restoration is an efficient means to speed up the restoration process. The system sectionalizing strategy consists of determining the proper splitting points to sectionalize the entire blackout area into several subsystems, so that parallel restoration can be carried out within the subsystems. For a large-scale power system, this system sectionalizing problem is quite complicated when considering black-start constraints, generation/load balance constraints, and voltage stability constraints. This paper presents an ordered binary decision diagram (OBDD) -based system sectionalizing method, by which the splitting points can be found quickly. Simulation results on the IEEE 118-bus test systems show that the method can successfully sectionalize the system into subsystems satisfying black-start constraints, generation/load balance constraints, and voltage stability constraints.
\end{abstract}

Index Terms-Ordered binary decision diagram, parallel restoration, sectionalizing of subsystems, transmission system restoration.

\section{INTRODUCTION}

$\mathbf{P}$ OWER system restoration involves a broad diversity of tasks. Among the numerous complicated factors, restoration of the system to normal operation as quickly as possible is the primary restoration objective. A centralized black-start capability could accomplish the cranking task to restore the entire system with a sequential restoration strategy. However, the time required to restore a large system by this method may be significantly longer. Hence, a parallel restoration method is commonly used by utilities in system restoration plans [1]. In the case of widespread blackouts, it is almost always advantageous to sectionalize the affected area into several subsystems to utilize parallel restoration of subsystems, and thus reduce restoration duration. Liu et al. suggest generic restoration actions (GRAs) [2]. These are termed: preparation, system restoration, and load restoration. The first stage is to partition a system into subsystems and send cranking power to non-black-start generators. The second and third stages are reintegration of the bulk power network and minimization of the impact of the outage by gradually picking up load.

The resynchronization of the subsystems is quite time consuming due to communication complexities and the lack of measurement data during the early stages of restoration. To bring

Manuscript received March 31, 2010; revised July 09, 2010; accepted August 30, 2010. Date of publication September 30, 2010; date of current version July 22, 2011. This work was supported by the Power System Engineering Research Center. Paper no. TPWRS-00253-2010.

C. Wang and V. Vittal are with the Department of Electrical Engineering, Arizona State University, AZ 85287 USA (e-mail: chong.wang.1 @ asu.edu; vijay. vittal@asu.edu).

K. Sun is with EPRI, Palo Alto, CA 94304 USA (e-mail: ksun@epri.com). Digital Object Identifier 10.1109/TPWRS.2010.2074216 the entire critical load to service and restore significant load as quickly as possible before the synchronization of subsystems, a methodology of sectionalizing strategy is required. This paper focuses on the optimized strategy for the first stage and introduces a three-step ordered binary decision diagram (OBDD)based search procedure, which can assist the system operator, by providing efficient sectionalizing strategies during restoration. The result can be provided in near real-time and is capable of handling unexpected system changes during restoration. A common parallel restoration sequence includes:

1) sectionalizing of power system into subsystems;

2) restoration of each subsystem;

3) synchronization of subsystems.

The determination of the parallel restoration sequence to be used in restoring a specific system is highly dependent on the system conditions. An inappropriate sectionalizing strategy can cause difficulty in synchronization of subsystems or cause restored load to be tripped again. Several criteria require to be verified before the parallel restoration determination in order to maintain system security and restoration reliability. These criteria include [1], [3]:

1) each subsystem must have at least one black-start generator;

2) each subsystem should match generation and load to maintain system frequency within prescribed limits;

3) system should have adequate voltage control capabilities to maintain a suitable voltage profile;

4) each subsystem should be monitored at the system control center to ensure its internal security.

In an effort to solve the problem taking into account the first three criteria above, a balanced partition problem (BP problem)based approach is considered [4]. The BP problem consists of determining proper strategies to ensure that the black-start generator constraint is satisfied, and the generation/load imbalance is limited. The BP problem has been proven to be NP-complete [4], i.e., no polynomial-time algorithm can solve it. The search space for solutions exponentially explodes with the growth in the number of transmission lines, whose on/off status is described by an equal number of Boolean variables. Since the BP problem contains a large number of Boolean variables corresponding to the transmission lines in a large power system, it is difficult and time consuming to determine all proper solutions. The OBDD-based sectionalizing approach can improve the solution efficiency of complex Boolean equations and has been widely used in the solution of other large partitioning problems [5], [6]. The OBDD-based methods have been previously applied in solving power system islanding strategies [7], [8].

This paper will focus on checking steady-state stability of the subsystems formed. After sectionalizing strategies are obtained 
by the proposed three-step method, transient stability can be checked by simulations on critical contingencies or line closing operations [9]. The sectionalizing strategies with all the parallel restoration constraints satisfied are candidates for the system dispatcher to consider in parallel restoration. After the search results are provided by the proposed method, the operator can select the best strategy based on updated system configuration and experience. Without loss of generality, only the case of sectionalizing one network into two subsystems is considered in the following sections. The approach can be extended to the more general case with more subsystems.

The rest of the paper is organized as follows. Section II introduces the BP problem and OBDD representation. In Section III, the approach to determine sectionalizing strategies in parallel restoration by an OBDD-based three-phase method is introduced. In Section IV, the method is applied to the IEEE 118-bus test networks to demonstrate its performance. Section $\mathrm{V}$ provides some concluding remarks. The OBDD algorithm and power balance error limit estimation method are provided in the Appendix.

\section{BP PROBLEM AND OBDD REPRESENTATION}

Not all generating plants are suitable candidates for blackstart units. During the parallel restoration process, all the available generators can be divided into two categories:

- Black-start generators-which are capable of providing cranking power to a de-energized power system.

- Non-black-start generators-which are incapable of providing power to a de-energized power system.

After the entire system is sectionalized into subsystems, each subsystem must have at least one black-start generator to provide power to non-black-start generators to carry out subsystem restoration. This constraint is denoted as a black-start constraint (BSC).

Each subsystem in the developed parallel restoration scheme should have the ability to match generation and load to a prescribed tolerance to maintain system frequency. Moreover, if the available generation level is close to the predicted load level inside each subsystem, a larger portion of the subsystem can be restored to facilitate the synchronization of subsystems. This constraint is denoted as the power balance constraint $(P B C)$.

When the subsystems are being synchronized, some open lines on the boundary of subsystems may cause system voltage problems. In the network related aspects, the critical lines that maintain system voltage stability should not be included on the sectionalizing boundary in the final sectionalizing strategy. This constraint is denoted as the voltage stability constraint (VSC).

The following BP problem consists of finding the sectionalizing strategies that satisfy $B S C$ and $P B C$ constraints identified above during parallel restoration.

If there are $N L$ branches in the power system, there are $2^{N L}$ possible branch state combinations to check $B S C$ and $P B C$ constraints. Hence, the BP problem of a large-scale power system is quite time consuming and complicated because a combinatorial explosion of its strategy space is unavoidable. Moreover, it is necessary to guarantee both correctness and speed in determining the final sectionalizing strategy to speed up restoration. The satisfiability of the Boolean expression of the sectionalizing strategy is developed and solved in Section III below. After the BP problem is solved, all the transmission lines should be evaluated to make sure no voltage violation occurs during subsystem synchronization.

The BP problem can be analytically stated as follows, given an undirected, connected and node-weighted graph $G(V, E, W)$, two subsets $V_{G A}, V_{G S}$ of $V$ and a positive constant $d$, search for a subset $E_{C}$ to split $G$ into two connected sub-graphs $G_{1}\left(V_{1}, E_{1}, W_{1}\right)$ and $G_{2}\left(V_{2}, E_{2}, W_{2}\right)$ such that $V_{G A} \subset V_{1}, V_{G S} \subset V_{2}(B S C)$ and the following constraint $(P B C)$ are satisfied:

$$
\left|\sum_{v_{i} \in V_{1}} W_{i}\right| \leq d, \quad\left|\sum_{v_{j} \in V_{2}} W_{j}\right| \leq d
$$

where

$$
\begin{array}{ll}
V=\left\{v_{1}, \ldots, v_{n}\right\} & \text { node set; } \\
d & \text { allowable power balance error tolerance; } \\
E & \text { branch set; } \\
W=\left\{w_{1}, \ldots, w_{n}\right\} & \text { weight set. }
\end{array}
$$

$w_{i}$ can be calculated by the following equation:

$$
W_{i}=S_{G}^{i}-S_{L}^{i}
$$

where $S_{G}^{i}$ is the injected complex generator power and $S_{L}^{i}$ is the complex load power at bus $i$. In this paper, the restoration paths inside the subsystems are not considered. Reactive power imbalance is highly related with energizing sequence. In the actual power network, the unbalanced reactive power can be compensated by local reactive power compensators, and the real power balance and real power flow are more important during restoration. So only the real power balance is considered, and the weight of node $i$ in the power network is defined in (3):

$$
W_{i}=P_{G}^{i}-P_{L}^{i}
$$

\section{OBDD-Based Three-Phase Sectionalizing Strategy}

In this section, the Boolean expression of $B S C$ and $P B C$ is first developed. An OBDD-based three-step search method is then introduced to solve the BP problem and maintain system voltage stability in power system parallel restoration. The three phases are described as follows.

\section{A. Step-1: Initialize OBDD Model Based on System Information}

To create an OBDD for a power system, every branch in the system can be seen as a Boolean variable, with respect to "0" or " 1 ". The branch with number " 0 " means this branch is open in the final sectionalizing strategy, and a branch with number " 1 " 


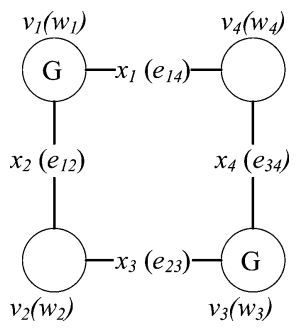

Fig. 1. Four-bus power system with two black-start units.

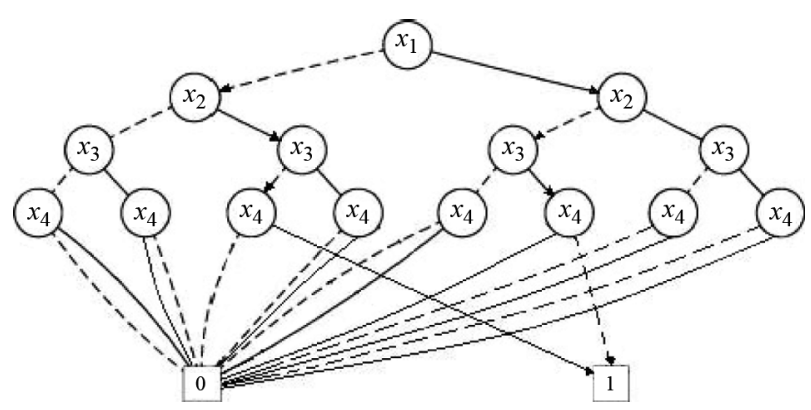

Fig. 2. Unreduced binary decision diagram for BSC in four-bus power system.

means it is closed in the final sectionalizing strategy. So each root node in the binary decision diagram represents one binary combination of all the branch states, which can be tracked by a bottom-up process.

All branches are then included in the binary decision diagram. For the different system constraints, if one binary combination of all the branch states satisfies all the constraints, then that root node will connect to the terminal node " 1 ". If any constraint violation occurs, then that root node will connect to the terminal node " 0 ". Using this logic, the binary decision diagram can be constructed with any branch order. For a specific power system, there is more than one OBDD with respect to different branch orders, but there is only one set of feasible solutions satisfying a given Boolean expression. For example, a four-bus system is shown in Fig. 1. It is assumed that both generators are black-start generators. To satisfy the $B S C$, two possible solutions are given: 1) $x_{1}, x_{3}$ are closed and $x_{2}, x_{4}$ are open; 2$) x_{1}, x_{3}$ are open and $x_{2}, x_{4}$ are closed. For these sets of possible solutions, the binary decision diagram is shown in Fig. 2.

When performing system restoration, operators need near real-time system information in order to make decisions under changing system conditions. Some information is extremely important in parallel restoration. These system conditions include:

- black-start capability;

- interconnection assistance;

- non-black-start plants status;

- status of transmission lines and breakers;

- predicted generation and load level inside each subsystem.

After the information is sent to the transmission operations center (TOC), the OBDD parameters are initialized. The blackstart unit and critical load are confirmed. The system load is assumed to be about $40 \%$ of the total available generator ratings. The binary variables corresponding to the lines with certain open or closed status are assigned values of " 0 " or " 1 ". System operators can also set the Boolean value based on their experience or special concern. This may eliminate some paths of the OBDD representing a feasible operation. The allowable power balance error tolerance $d$ is also chosen by the TOC. Then the binary decision diagram is maximally reduced by applying the two reduction rules in OBDD. (These reduction rules are described in the Appendix.)

The feasible sectionalizing strategy can then be searched in the OBDD. The procedure to build the Boolean expression including both $B S C$ and $P B C$ is introduced in Step- 2 .

\section{B. Step-2: Building OBDD and Solving BP Problem}

Before the Boolean expressions of $B S C$ and $P B C$ are presented, the concept of the adjacency matrix of an $N$-node network $G(V, E, W)$ is introduced. The elements $A_{G_{i j}}$ and $A_{G_{j i}}$ of the adjacency matrix $A_{G}$ are the same Boolean variable, denoted by $b_{i j}$. If there is an edge $e_{i j} \in E, A_{G_{i j}}=A_{G_{j i}}=b_{i j}=$ 1 ; otherwise, $A_{G_{i j}}=A_{G_{j i}}=0$. For example, the $A_{G}$ of the four-bus power system shown in Fig. 1 is given by (4):

$$
A_{G}=\left[\begin{array}{cccc}
0 & b_{12} & 0 & b_{14} \\
b_{12} & 0 & b_{23} & 0 \\
0 & b_{23} & 0 & b_{34} \\
b_{14} & 0 & b_{34} & 0
\end{array}\right]
$$

It is easy to draw the following conclusions from Boolean matrix theory:

$$
A_{G}^{*} \triangleq I \oplus A_{G}^{1} \oplus A_{G}^{2} \oplus \cdots \oplus A_{G}^{L}
$$

where $L$ is the longest path in $G(V, E, W)$ in terms of the number of branches between two arbitrary nodes in the network. $I$ is identity matrix ( 1 on the diagonal and 0 elsewhere) which has the same dimension as $A_{G}$, and $A_{G}^{i}$ is the matrix $A_{G}$ raised to the $i$ th power. The symbol " $\oplus$ " here represents the "OR" operation on each element of the matrices. Then $A_{G}^{*}$ can determine the connection of any arbitrary pair of nodes inside the network. If $A_{G_{i j}}^{*}=1$, there must exist a connection path between bus $i$ and bus $j$.

For example, considering the four-bus power system, $L=3$ and all the paths from $v_{1}$ to $v_{4}$ in Fig. 1 can be obtained by (6):

$$
A_{G_{14}}^{*}=b_{14} \otimes b_{12} \otimes b_{23} \otimes b_{34}
$$

where $b_{14}$ and $b_{12} \otimes b_{23} \otimes b_{34}$ correspond with paths $e_{14}$ and $e_{12} e_{23} e_{34}$. If the system is sectionalized into two subsystems by opening branch $e_{12}$ and $e_{34}, A_{G_{14}}^{*}=b_{14}=1$ by setting $b_{12}=$ $b_{34}=0$. This represents that bus 1 and bus 4 are connected in the above sectionalizing strategy.

Then the Boolean expression of $B S C$ and $P B C$ can be built from $A_{G}^{*}$. For the convenience of expression, two sets of generator groups are defined as $V_{A}^{G}$ and $V_{S}^{G}$. There is at least one 
black-start generator in each group. Three sets of serial numbers of the nodes in the network are defined:

$$
\begin{aligned}
I_{A}=\left\{i \mid v_{i} \in V_{A}^{G}\right\}, & I_{S}=\left\{j \mid v_{j} \in V_{S}^{G}\right\} \\
\text { and } & I_{G}=I_{A} \cup I_{S} .
\end{aligned}
$$

$I_{G}$ contains the serial numbers of all generator nodes. Select any two arbitrary elements $i_{A} \in I_{A}$ and $i_{S} \in I_{S}$. Then, $B S C$ can be expressed as

$B S C=\prod_{i \in I_{A}}^{\otimes} A_{G_{i, i_{A}}}^{*} \otimes \prod_{j \in I_{S}}^{\otimes} A_{G_{j, i_{S}}}^{*} \otimes \prod_{k \notin I_{G}}^{\otimes}\left(A_{G_{k, i_{A}}}^{*} \bar{\oplus} A_{G_{k, i_{S}}}^{*}\right)$

where " $\overline{\oplus "}$ "represents the "EXCLUSIVE-OR" operation. The first two product terms guarantee that all the generators inside each subsystem are connected to each other. The third product term guarantees that each load bus connects to only one blackstart generator. Obviously, BSC is a Boolean function of all $b_{i j}$ 's.

Then the $P B C$ can be expressed as

$$
P B C=\prod_{i=1-N}^{\otimes}\left\langle\left|A_{G_{i^{*}}}^{*} \times W\right|<d\right\rangle
$$

where $A_{G_{i} *}^{*}$ is the $i$ th row of $A_{G}^{*}$ and $W=\left[w_{1}, \ldots, w_{n}\right]^{T}$ is the real power weight vector of $N$ nodes, which has been introduced in Section II-A. $A_{G_{i^{*}}}^{*} \times W$ is the real power balance error in the subsystem that contains node number $i$ in the network. Therefore, the product guarantees that the real power balance in each subsystem is smaller than the power balance error tolerance $d$. The Appendix lists the power balance error tolerance $d$ estimation method.

Variable orderings of $b_{i j}$ are selected based on the node weights. The branch with the larger sum of the two ending nodes weights of that branch has a higher order. This ordering is more efficient than the random orderings. Then the OBDDs of $B S C$ and $P B C$ can be developed and simplified using the OBDD software packages. Finally, the OBDD of the BP problem is obtained by applying operation "AND" on BSC and $P B C$ expressions:

$$
B P=B S C \otimes P B C
$$

\section{Step-3: Identifying Voltage Critical Tie-Lines}

During the synchronization of the subsystems, the open boundary tie-lines can be considered as outaged lines before they are closed. To identify critical lines that cannot lie at the sectionalizing boundary, $N-1$ voltage stability analysis is conducted for all line outages in the system. The most severe outages are selected using the contingency screening method available in VSAT [10].

The contingency screening feature in VSAT is designed to identify the critical contingencies. The screening of critical line contingencies classifies the contingencies based on their voltage

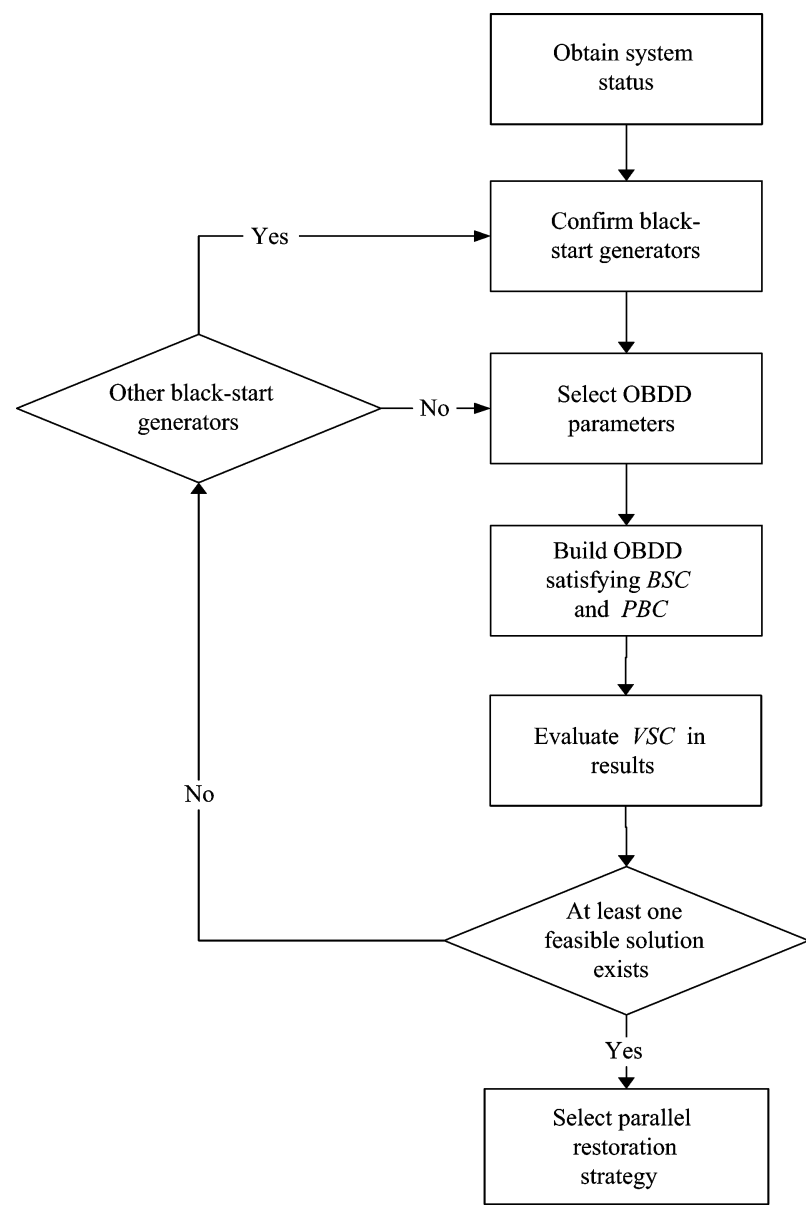

Fig. 3. Parallel restoration strategies searching method flow chart.

stability margin. The voltage stability margin of each contingency is defined as the difference between the pre-contingency transfer at the initial operating point and the last point where the post-contingency solution exists [10].

In this phase, all the sectionalizing strategies from Step-2 are evaluated based on their voltage stability performance. The candidate strategies with critical lines on the boundary will be eliminated. Moreover, the voltage stability margin of each contingency inside the subsystems can be obtained as needed.

Fig. 3 shows the flow chart of the proposed three-step OBDDbased search procedure.

\section{Simulation Results}

In this section, the standard IEEE 118-bus data are used to verify the proposed method. There are 186 branches in the network. To accelerate the search, a network simplification method introduced in [8] can be used. In this paper, the network simplification is not considered and the original network is used. The generation/load mismatch limit is chosen as $d=150 \mathrm{MW}$. The real-power generator data are shown in Table I. Several assumptions are made before the BP problem is solved using the OBDD algorithm. Bus 31 and bus 87 are assumed as black-start generators and the cranking groups are given in Table II. The selection of the cranking groups is based on the generators synchronization groups in contingency analysis when their synchronization 


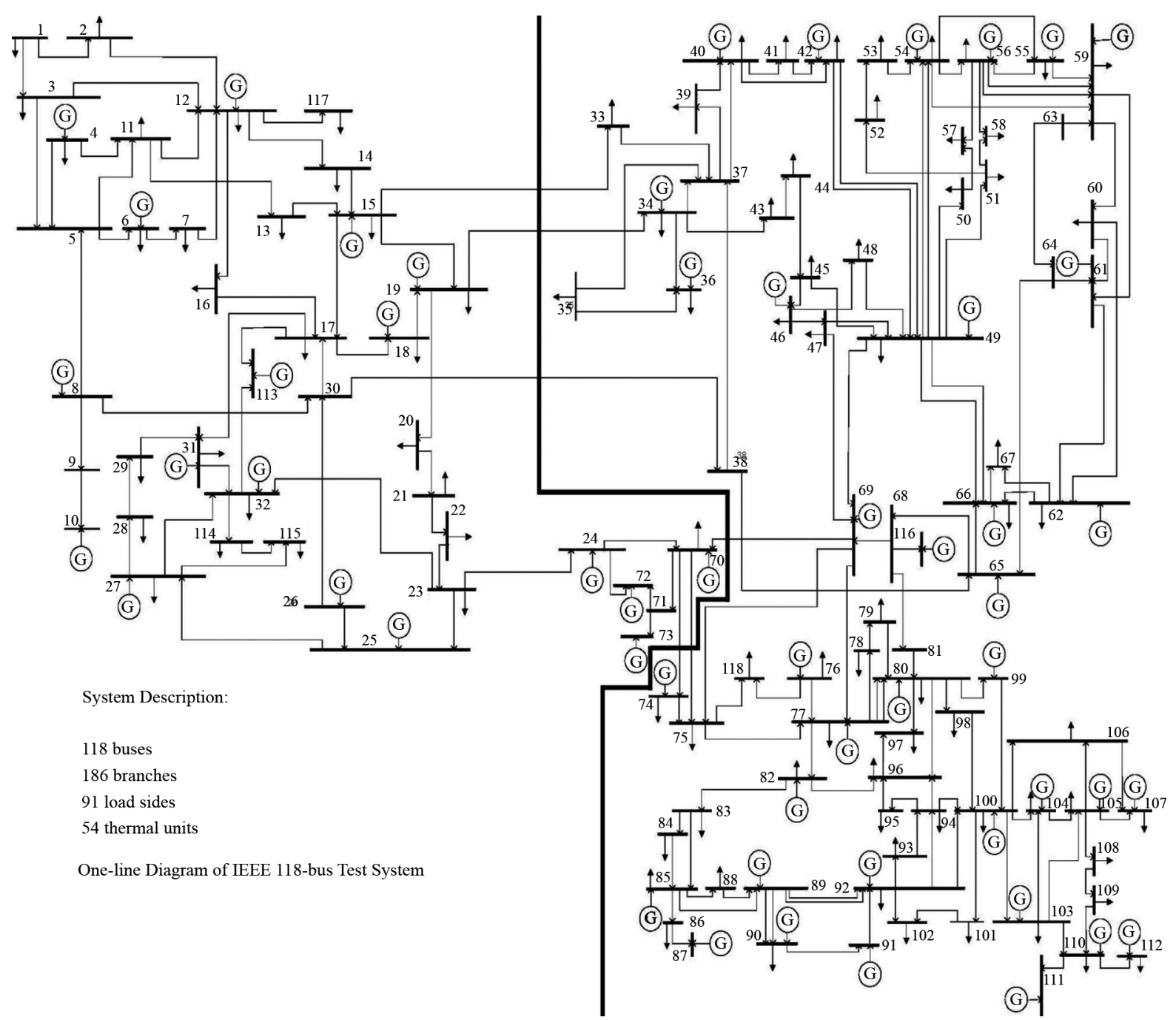

Fig. 4. Sectionalizing strategy on IEEE 118-bus system.

TABLE I

GENERATOR DATA OF IEEE 118-BUS SYSTEM

\begin{tabular}{c|c|c|c|c|c}
\hline Bus No. & $\mathrm{P}_{\mathrm{G}}(\mathrm{MW})$ & Bus No. & $\mathrm{P}_{\mathrm{G}}(\mathrm{MW})$ & Bus No. & $\mathrm{P}_{\mathrm{G}}(\mathrm{MW})$ \\
\hline 10 & 180.00 & 54 & 19.20 & 87 & 1.60 \\
\hline 12 & 34.00 & 59 & 62.00 & 89 & 242.80 \\
\hline 25 & 88.00 & 61 & 64.00 & 100 & 100.80 \\
\hline 26 & 125.60 & 65 & 156.40 & 103 & 16.00 \\
\hline 31 & 2.80 & 66 & 156.80 & 111 & 14.40 \\
\hline 46 & 7.60 & 69 & 206.40 & & \\
\hline 49 & 41.60 & 80 & 190.80 & & \\
\hline
\end{tabular}

TABLE II

Generator CRANKING Groups of IEEE 118-Bus SYSTEM

\begin{tabular}{c|c|c}
\hline Group No. & Node Serial Numbers & $\begin{array}{r}\text { Total real power } \\
\text { generation (MW) }\end{array}$ \\
\hline 1 & $\mathbf{3 1}, 10,12,25,26$ & 431 \\
\hline 2 & $\begin{array}{c}\mathbf{8 7}, 46,49,54,59,61,65,66, \\
69,80,89,100,103,111\end{array}$ & 1274 \\
\hline
\end{tabular}

is lost [7]. The generation level is reduced to about $40 \%$ of generation ratings with the entire critical load in service.

There are 211 possible sectionalizing strategies. The results for the critical contingency screening based on voltage performance are shown in Table III based on the maximum power
TABLE III

Voltage Stability ScREenING ON IEEE 118- Bus System

\begin{tabular}{l|c|c|c|r}
\hline Rank & Type & $\begin{array}{c}\text { From Bus } \\
\text { Number }\end{array}$ & $\begin{array}{c}\text { To Bus } \\
\text { Number }\end{array}$ & Zone \\
\hline 1 & Line & 8 & 9 & 1 \\
\hline 2 & Line & 9 & 10 & 1 \\
\hline 3 & Line & 38 & 65 & 1 \\
\hline 4 & Line & 8 & 5 & 1 \\
\hline 5 & Line & 38 & 37 & 1 \\
\hline
\end{tabular}

transfer level. After eliminating the candidate strategies which contain these voltage stability vulnerable lines, there are 137 strategies that satisfy $B S C, P B C$, and VSC. Fig. 4 shows one possible system sectionalizing strategy. Six lines are open in the sectionalizing strategy; they are

$$
\left\{e_{15,33}, e_{19,34}, e_{30,38}, e_{69,70}, e_{70,74}, e_{70,75}\right\} .
$$

Simulation results reveal that for a graph with a manageable number of Boolean variables, the BP problem can be solved in a short time. The method can be efficiently used to search for 
power system parallel restoration sectionalizing strategies. In fact, a large power system may have more than two black-start units. The proposed method can be user defined and facilitate further sectionalizing.

The BuDDy package (v2.0) [11], which supports all standard OBDD operations and especially many highly efficient OBDD vector operations, is selected to develop a program in $\mathrm{C}++$ language on a PC (Core2 6700-2.66G CPU and 2.0GB DDRAM). The package has evolved from a simple introduction to OBDDs to all the standard OBDD operations.

With the black-start constraint and generation/load constraint checking, the total search time on a 186-branch system is less than 3 min.

These results reveal that the checking module found two solutions for the BP problem. In the actual case, once it is determined that parallel restoration is feasible for the system, the operators only need to consider the selected candidate strategies instead of all $2^{186}$ strategies. This is a very big saving in time. The operators' final decision will be made with consideration of the current state of system and availability of alternative generators and lines. If none of the sectionalizing strategies are suitable for the current system status, the operator can change the generation cranking group or relieve power balance error tolerance for further searching.

It should be noted that the generators' cranking group can be different. To maximize the generation capability, all the non-black-start generators are considered available during the restoration. The problem of optimizing generation capability during the restoration is complex and involves a large number of combinatorial choices [12]. A number of constraints have to be verified before initiating the cranking process. The constraints mainly include voltage and frequency transient, voltage drop, and protective relay actions[13]. Hence, the black-start and non-black-start generators start-up sequence should be adjusted depending on real-time generator status. Moreover, from the network topology, it is observed that arbitrary generator groups cannot guarantee that there is a sectionalizing strategy. In the actual case, the operators have to evaluate the generator groups considering these constraints and cranking sequence. If the system does not have enough generation capability in each subsystem, the load level in the subsystems should be reduced. Before all the generating units are restored or the subsystems are synchronized, operators should maintain the generation/load balance in the subsystems.

\section{CONCLUSIONS}

This paper presents an efficient approach to search for system sectionalizing strategies using the OBDD-based technology. The proposed method decomposes the splitting search process into three parts: 1) determination of branch status and ordering in OBDD, 2) finding the boundary of the subsystems under the black-start constraint and constraint of minimizing the generation/load imbalance error, and 3) evaluation of system voltage stability during parallel restoration.

The proposed approach will sectionalize the system into suitable islands or subsystems that would facilitate parallel restoration. Then a sequential restoration would accomplish the restoration in each subsystem. After the subsystems are restored, the tie-lines could be energized to synchronize subsystems. The method was tested on the IEEE 118-bus test networks. The results indicate that method is effective during the early stages of the restoration as they provide guidance to the operators on how parallel restoration could be performed.

The method focuses on the first stage of the restoration. It gives the system operators direct guidance with respect to sectionalizing strategy and generator cranking groups. The results can be provided in real time after system condition change. This three-step method may not be the final sectionalizing strategy. In addition, there is no need to stick to the rigid boundary of the subsystems. The OBDD variable values can be updated with the unpredicted system condition changes or specified by operators. This paper provides a search method that augments the operators' capabilities. The constraints can also be updated and checked with operator choices after OBDD searching.

\section{APPENDIX A \\ BP PROBLEM AND OBDD REPRESENTATION}

It has been proved that the problem of determining satisfiability of Boolean expressions is NP-complete [14]. This check of satisfiability involves the determination of whether the Boolean expression has value " 0 " or "1". An OBDD [15] is a directed acyclic graph (DAG) representation of a Boolean expression and is commonly used to determine satisfiability of Boolean expressions. The time complexity of checking the satisfiability of a Boolean function $f\left(x_{1}, \ldots, x_{n}\right)$ is $O(n)$. Once the OBDD of a function is built, its satisfiability can be verified in polynomial time. But the choice of variable ordering of $x_{1}, \ldots, x_{n}$ cannot be solved in polynomial time and has significant impact on the size of the OBDD. Once the variable ordering has been chosen carefully, different kinds of logic operations can be performed efficiently on the OBDD.

Fig. 5 shows an OBDD (the reduction rules have not been applied), representing the function $f\left(x_{1}, x_{2}, x_{3}\right)=x_{1} \otimes x_{2} \otimes$ $x_{3}$, where the symbol " $\otimes$ " represents the "AND" operation, and the symbol " $\oplus$ " represents the "OR" operation. In the OBDD, a variable $x_{i}$ with a "False" value (having value " 0 ") is denoted by a dashed line and the variable $x_{i}$ with a "True" value (having value " 1 ") is denoted by a solid line. Once all possible values of a variable have been addressed, the OBDD progresses to the next level at which the states of the next variable are addressed. As can be seen from Fig. 5, the complete OBDD is complex and can be reduced by applying the following two reduction rules [15], [16]:

1) merging rule-two isomorphic subgraphs should be merged. As shown in Fig. 6(a), $v_{1}$ and $v_{2}$ connect to the same nodes and they both either have value " 0 " or " 1 ". Then $v_{1}$ and $v_{2}$ should be merged into a new node $v_{3}$. All nodes that point to $v_{1}$ or $v_{2}$ are connected to $v_{3}$ after reduction.

2) deletion rule-a node whose two branches point to the same node should be deleted. As shown in Fig. 6(b), both branches of $v_{4}$ point to the same node irrespective of whether $v_{4}$ has value " 0 " or " 1 ". Then $v_{4}$ should be deleted. All nodes pointing to $v_{4}$ in the upper level are 


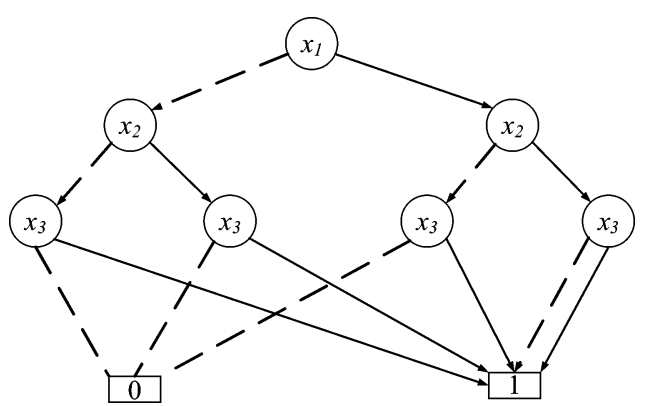

Fig. 5. Binary decision diagram representing the function $f\left(x_{1}, x_{2}, x_{3}\right)=$ $x_{1} \otimes x_{2} \oplus x_{3}$.

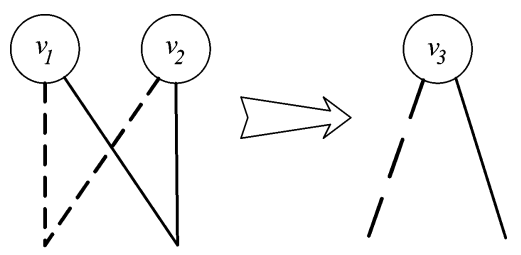

(a)

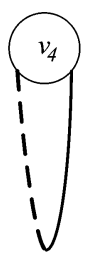

(b)

Fig. 6. OBDD reduction rules. (a) Merging rule. (b) Deletion rule.

directly connected to the node that $v_{4}$ pointed to before in the lower level.

Applying these rules for reduction, Fig. 7 shows the reduced OBDD, from which it is straightforward to get the solution to the satisfiability of Boolean expressions problem depicted in Fig. 7.

There are three paths, associated with five possible solutions, and the three paths are marked using arrows in Fig. 7. The result corresponding to each path is shown in Table IV. In the table, an "X" associated with a variable means that irrespective of whether the variable $x_{i}$ is "True" or "False" in that path, the OBDD checking result will remain the same.

\section{APPENDIX B}

\section{POWER BALANCE ERror Estimation}

To find an estimate of $d$, a reduced model of a reheat unit for frequency decline analysis [17], [18] is used. According to the North East Power Coordinating Council (NPCC) standard, the automatic under-frequency load shedding (UFLS) should be deployed immediately if system frequency drops to $57 \mathrm{~Hz}$ [19].

Typical system data are used to compute the minimum power balance error that can drive the system to the frequency of 57 $\mathrm{Hz}$ for each subsystem restored in parallel. This gives the power balance error tolerance. For a typical reheat turbine model ne-

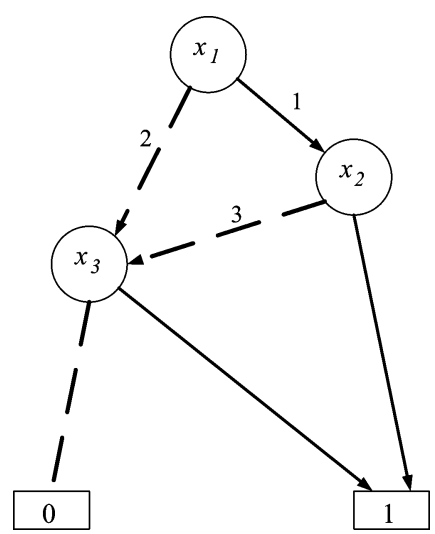

Fig. 7. Reduced ordered binary decision diagram representing the function $f\left(x_{1}, x_{2}, x_{3}\right)=x_{1} \otimes x_{2} \oplus x_{3}$.

TABLE IV

RESULT OF OBDD REDUCTION

\begin{tabular}{c|c|c|c}
\hline Path & $x_{1}$ & $x_{2}$ & $x_{3}$ \\
\hline 1 & 1 & 1 & $\mathrm{X}$ \\
\hline 2 & 0 & $\mathrm{X}$ & 1 \\
\hline 3 & 1 & 0 & 1 \\
\hline
\end{tabular}

glecting all smaller time constants, the speed deviation can be expressed as

$$
\Delta \omega=\left(\frac{R \Omega_{n}^{2}}{D R+K_{m}}\right)\left(\frac{\left(1+T_{R} S\right) P_{d}}{S^{2}+2 \Omega_{n}^{2} \lambda S+\Omega_{n}^{2}}\right)
$$

where

$$
\begin{aligned}
\Omega_{n}^{2} & =\frac{D R+K_{m}}{2 H R T_{R}} \\
\lambda & =\left(\frac{2 H R+\left(D R+K_{m} f_{H}\right) T_{R}}{2\left(D R+K_{m}\right)}\right) \Omega_{n} .
\end{aligned}
$$

Here $K_{m}$ is a mechanical power gain factor. A typical value of 0.95 is used.
$H \quad$ inertia constant in seconds, typically $4.0 \mathrm{~s}$;
$f_{H} \quad$ high-pressure power fraction, typically 0.3 ;
$D \quad$ damping factor, typically 1.0 ;
$T_{R} \quad$ reheat time constant, seconds, typically $8.0 \mathrm{~s}$;
$R \quad$ fraction of the reheat turbine, typically 0.059 ;
$P_{d} \quad$ power balance error, in per unit'
$P_{\text {sys }} \quad$ real power in entire system;
$P_{\text {subsys }}$ real power in subsystem.

Usually $P_{d}$ is in the form of a step function. The time domain solution for the speed deviation can be directly obtained from (11) as follows:

$$
\begin{aligned}
P_{d} & =P_{\text {step }} u(t) \\
\Delta \omega & =\left(\frac{R P_{\text {step }}}{D R+K_{m}}\right)\left(1+a e^{-\lambda \omega_{n} t} \sin \left(\omega_{r} t+\phi\right)\right)
\end{aligned}
$$




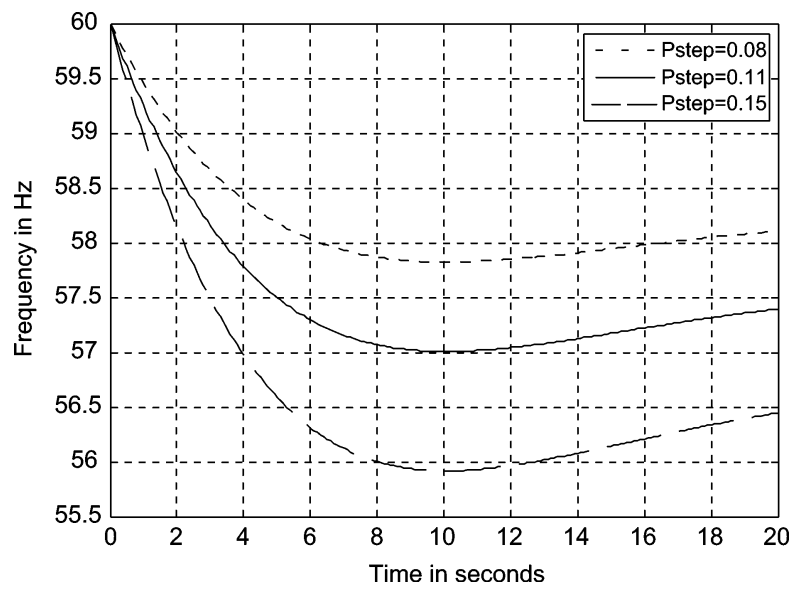

Fig. 8. Frequency response to different power balance error value.

where

$$
\begin{aligned}
a & =\sqrt{\frac{1-2 T_{R} \lambda \omega_{n}+T_{R}^{2} \omega_{n}^{2}}{1-\lambda^{2}}} \\
\omega_{r} & =\omega_{n} \sqrt{1-\lambda^{2}} \\
\phi & =\phi_{1}-\phi_{2} \\
\phi_{1} & =\tan ^{-1}\left(\frac{\omega_{r} T_{R}}{1-\lambda \omega_{n} T_{R}}\right) \\
\phi_{2} & =\tan ^{-1}\left(\frac{\sqrt{1-\lambda^{2}}}{-\lambda}\right) .
\end{aligned}
$$

Using this reduced model and normalizing, the lowest system average frequency for this disturbance is obtained as $57 \mathrm{~Hz}$ when $P_{d}=0.11 P_{\text {sys. }}$. So, $0.11 P_{\text {subsys }}$ is chosen as the power balance error limit in each subsystem. A lower $d$ value is selected to improve system frequency response during load restoration procedure based on system conditions. The system frequency deviation for different values of power balance error is shown in Fig. 8.

\section{ACKNOWLEDGMENT}

The authors would like to thank Dr. J. Lind-Nielsen for his help in using the BuDDy package.

\section{REFERENCES}

[1] M. M. Adibi, P. Celland, L. H. Fink, H. Happ, R. J. Kafka, D. Scheurer, and F. Trefny, "Power system restoration-A task force report," IEEE Trans. Power Syst., vol. 2, no. 2, pp. 271-277, May 1987.

[2] L. H. Fink, K. L. Liou, and C. C. Liu, "From generic restoration actions to specific restoration strategies," IEEE Trans. Power Syst., vol. 10, no. 2, pp. 745-751, May 1995.
[3] M. M. Adibi, R. W. Alexander, and B. Avramovic, "Overvoltage control during restoration," IEEE Trans. Power Syst., vol. 7, no. 4, pp. 1464-1470, Nov. 1992.

[4] Q. Zhao et al., "A study of system splitting strategies for island operation of power system: A two-phase method based on OBDDs," IEEE Trans. Power Syst., vol. 18, no. 4, pp. 1556-1565, Nov. 2003.

[5] V. Raman and A. N. Zamfirescu, "OBDD extraction from VHDL gate level descriptions at design elaboration," in Proc. Fall VHDL Int. Users Forum Workshop, 1999, pp. 30-39.

[6] F.-M. Yeh and S.-Y. Kuo, "OBDD-based network reliability calculation," Electron. Lett., vol. 33, pp. 759-760, Apr. 1997.

[7] K. Sun, D. Zheng, and Q. Lu, "Searching for feasible splitting strategies of controlled system islanding," Proc. Inst. Elect. Eng., Gen., Transm., Distrib., vol. 153, no. 1, pp. 89-98, Jan. 2006.

[8] K. Sun, D. Zheng, and Q. Lu, "Splitting strategies for islanding operation of large-scale power systems using OBDD-based methods," IEEE Trans. Power Syst, vol. 18, no. 2, pp. 912-923, May 2003.

[9] C. Wang, V. Vittal, V. S. Kolluri, and S. Mandal, "PTDF-based automatic restoration path selection," IEEE Trans. Power Syst., to be published.

[10] DSATools, Surrey, BC, Canada, Powertech Labs Inc.

[11] Jørn Lind-Nielsen's BuDDy Package. [Online]. Available: http:// sourceforge.net/projects/buddy/develop.

[12] R. D. Shultz and G. A. Mason, "Blackstart utilization of remote combustion turbines, analytical analysis and field test," IEEE Trans. Power App. Syst., vol. PAS-103, no. 8, pp. 2186-2191, Aug. 1984.

[13] M. M. Adibi, D. P. Milanicz, and T. L. Volkmann, "Remote cranking of steam electric station," IEEE Trans. Power Syst., vol. 11, no. 3, pp. 1613-1618, Aug. 1996.

[14] C. H. Papadimitriou, Combinatorial Optimization: Algorithms and Complexity. Englewood Cliffs, NJ: Prentice-Hall, 1982.

[15] R. E. Bryant, "Graph-based algorithms for Boolean function manipulation," IEEE Trans. Comput., vol. C-35, pp. 677-691, Aug. 1986.

[16] H.-T. Liaw and C.-S. Lin, "On the OBDD-representation of general Boolean functions," IEEE Trans. Comput., vol. 41, no. 6, pp. 661-664, Jun. 1992.

[17] P. M. Anderson and M. Mirheydar, "An adaptive method for setting underfrequency load shedding relays," IEEE Trans. Power Syst., vol. 7, no. 2, pp. 720-729, May 1992.

[18] H. You, V. Vittal, and Z. Yang, "Self-healing in power systems: an approach using islanding and rate of frequency decline-based load shedding," IEEE Trans. Power Syst., vol. 18, no. 1, pp. 174-181, Feb. 2003.

[19] NPCC Emergency Operation Criteria. [Online]. Available: http:// www.npcc.org/viewDoc.aspx?name=PRC-006-NPCC-01_082709. pdf\&cat=openProcess.

Chong Wang (S'09) received the B.E. degree in electrical engineering from Tsinghua University, Beijing, China, in 2006. He is currently pursuing the Ph.D. degree in the Electrical Engineering Department at Arizona State University, Tempe.

Vijay Vittal (S'78-F'97) received the B.E. degree in electrical engineering from the B.M.S. College of Engineering, Bangalore, India, in 1977, the M.Tech. degree from the Indian Institute of Technology, Kanpur, India, in 1979, and the Ph.D. degree from Iowa State University, Ames, in 1982

Dr. Vittal is a member of the National Academy of Engineering.

Kai Sun (M'06) received the B.S. degree in automation and the Ph.D. degree in control science and engineering from Tsinghua University, Beijing, China, in 1999 and 2004, respectively.

He was a postdoctoral research associate at Arizona State University, Tempe, from 2005 to 2007. He is currently a project manager at ERPI, Palo Alto, CA. 